Projets

de paysage

\section{Projets de paysage}

Revue scientifique sur la conception et l'aménagement de l'espace

13 | 2015

Biodiversité et paysage

\title{
Les marges sociales et les franges agricoles se tournent-elles le dos?
}

Une analyse des paysages alimentaires dans le quartier des Minguettes, Vénissieux

Do Suburban Areas and Agricultural Fringes Turn their Backs on One Another?

- A Study of Food Producing Landscapes in the Minguettes District of Vénissieux

\section{Alice Nikolli, Julie Le Gall et Myriam Laval}

\section{(2) OpenEdition}

\section{Journals}

Édition électronique

URL : http://journals.openedition.org/paysage/10182

DOI : $10.4000 /$ paysage. 10182

ISSN : 1969-6124

Éditeur :

École nationale supérieure du paysage de Versailles-Marseille, Institut national des sciences appliquées Centre Val de Loire - École de la nature et du paysage, École nationale supérieure d'architecture et de paysage de Bordeaux, École nationale supérieure d'architecture et de paysage de Lille, Agrocampus Angers

\section{Référence électronique}

Alice Nikolli, Julie Le Gall et Myriam Laval, « Les marges sociales et les franges agricoles se tournentelles le dos ? », Projets de paysage [En ligne], 13 | 2015, mis en ligne le 31 décembre 2015, consulté le 04 septembre 2020. URL : http://journals.openedition.org/paysage/10182 ; DOI : https://doi.org/ 10.4000/paysage.10182

Ce document a été généré automatiquement le 4 septembre 2020

Projets de paysage 


\section{Les marges sociales et les franges agricoles se tournent-elles le dos?}

Une analyse des paysages alimentaires dans le quartier des Minguettes, Vénissieux

Do Suburban Areas and Agricultural Fringes Turn their Backs on One Another? - A Study of Food Producing Landscapes in the Minguettes District of Vénissieux

Alice Nikolli, Julie Le Gall et Myriam Laval

\section{NOTE DE L'AUTEUR}

Ce travail est issu du mémoire de master 1 d'Alice Nikolli, « L'accès aux produits de l'agriculture locale dans un quartier de banlieue lyonnaise : l'exemple des Minguettes (Vénissieux) », ENS de Lyon, 2014, 150 p. Il participe également du programme de recherche émergent Marguerite financé par l'ENS de Lyon et réalisé au sein de l'UMR 5600 Environnement Ville Société.

1 Dans le contexte de la multiplication des crises sanitaires et de la remise en cause de l'agriculture productiviste, on remarque une promotion particulière des circuits courts, entendus comme des circuits de distribution des produits agricoles comptant au maximum un intermédiaire (Barnier, 2009). Ils sont notamment encouragés par les pouvoirs publics, à travers le plan Barnier de 2009 et la loi de modernisation agricole de 2010 (Beraud-Sudreau, 2010) et viennent modifier la relation des consommateurs urbains aux produits agricoles locaux.

Cependant, tous les quartiers des villes sont-ils concernés au même titre par cet engouement pour les circuits courts? Alors que les initiatives qui connectent les "petits producteurs locaux » aux marchés urbains font désormais partie du quotidien des populations à fort capital social et culturel, on constate que les marges sociospatiales urbaines - les quartiers défavorisés, caractérisés par une forte précarité socioéconomique - restent souvent à l'écart de ces nouveaux modes 
d'approvisionnement (Alkon et Agyeman, 2011) malgré le déploiement d'initiatives récentes (réseau Andes, épiceries mobiles, associations locales...). Pourquoi les marges urbaines et les franges agricoles se tournent-elles le dos? Plusieurs études indiquent que le prix des produits n'est pas la seule barrière pour que la commercialisation et la consommation de proximité s'ouvrent à des milieux sociaux variés (Mundler, 2013 ; Rödiger et Hamm, 2015). L'objectif de cet article est donc de tester un autre facteur susceptible d'expliquer cette mise à l'écart: les représentations que se font les habitants issus de ces marges sociospatiales de leur offre alimentaire et de l'agriculture locale. Les représentations des consommateurs et des producteurs en matière d'approvisionnement et d'alimentation s'expriment en effet par des choix et des pratiques susceptibles d'influencer les configurations des systèmes alimentaires (Dixon et Isaacs, 2013).

3 En ce sens, nous convoquons de façon exploratoire la notion de «paysage alimentaire ». Le paysage, comme portion d'espace soumis à la vue, est un construit social qui comporte à la fois des aspects matériels et immatériels et permet donc de saisir les représentations de l'individu qui l'observe. La relation entre paysage et alimentation est cependant encore peu travaillée par les sciences sociales. En anglais, on trouve quelques occurrences de l'expression food landscape dont la définition varie selon les travaux concernés : on peut citer l'agrifood landscape (Allen et al., 2003), désignant le système agroalimentaire en général, ou encore le commercial food landscape (Kelly et al., 2008), renvoyant à l'ensemble des commerces auquel un groupe d'acteurs a accès en fonction de sa localisation. En français, entretien des paysages ruraux et production alimentaire sont considérés comme deux des fonctions de l'agriculture, participant à sa multifonctionnalité (Jouve et Padilla, 2007), mais le "paysage alimentaire » n'est pas conceptualisé en tant que tel. La notion recouvrirait ce que connaissent les acteurs de leur environnement alimentaire et la façon dont ils se le représentent. Elle donne des indications sur la diversité des perceptions de l'offre alimentaire locale. Elle pourrait ainsi éclairer les choix des consommateurs, des producteurs, des acteurs publics en matière alimentaire, de même que les connexions ou déconnexions qui en découlent entre des espaces urbains et leurs franges agricoles.

4 Étudier les marges urbaines, au sens spatial et social, permet de sortir des territoires habituellement étudiés au sujet des circuits courts dans la recherche française - à savoir des quartiers centraux, concentrant des classes moyennes et aisées à fort capital social et culturel - dans l'optique d'une réflexion sur la justice alimentaire. La justice alimentaire vise à assurer "un partage équitable des bénéfices et des risques concernant les lieux, les produits et la façon dont la nourriture est produite et transformée, transportée et distribuée, et accessible et mangée » (Gottlieb et Joshi, 2010). Sur le plan alimentaire, ce sont les marges urbaines qui sont supposées « souffrir le plus des injustices du système alimentaire global » (Paddeu, 2012). Ainsi, les travaux de recherche dans lesquels la notion de food justice a été développée, menés aux ÉtatsUnis et au Canada notamment, s'intéressent en premier lieu à des marges sociospatiales urbaines: des quartiers défavorisés, concentrant les minorités sociales et ethniques (Block et al., 2004 ; Larsen \& Gilliland, 2008 ; Paddeu, 2012).

5 Après avoir décrit notre terrain d'étude et son offre alimentaire et agri-alimentaire, nous présenterons notre protocole pour analyser les paysages alimentaires des différents acteurs du quartier. Les résultats de ce travail permettent d'établir ensuite une typologie des paysages alimentaires, en fonction des représentations des acteurs 
considérés, et de mieux comprendre les connexions et déconnexions avec l'agriculture locale.

\section{Le quartier des Minguettes, un désert du circuit court}

Notre choix d'une marge sociale et spatiale pour cerner les paysages alimentaires des acteurs et leur relation à l'agriculture proche s'est porté sur le quartier des Minguettes, dans la commune de Vénissieux, au sein de l'agglomération lyonnaise (figure 1). Il s'agit d'un quartier de grands ensembles construit entre 1963 et 1973 sur un plateau qui était jusqu'alors agricole, et qui compte environ 20000 habitants. Ce quartier a été de longue date une cible de la politique de la ville, car il est marqué par une forte précarité économique et sociale : en $2009,30 \%$ de la population fait partie de la catégorie « bas revenu ", tandis que $78 \%$ a un niveau scolaire inférieur au baccalauréat.

Figure 1. Localisation du quartier d'étude

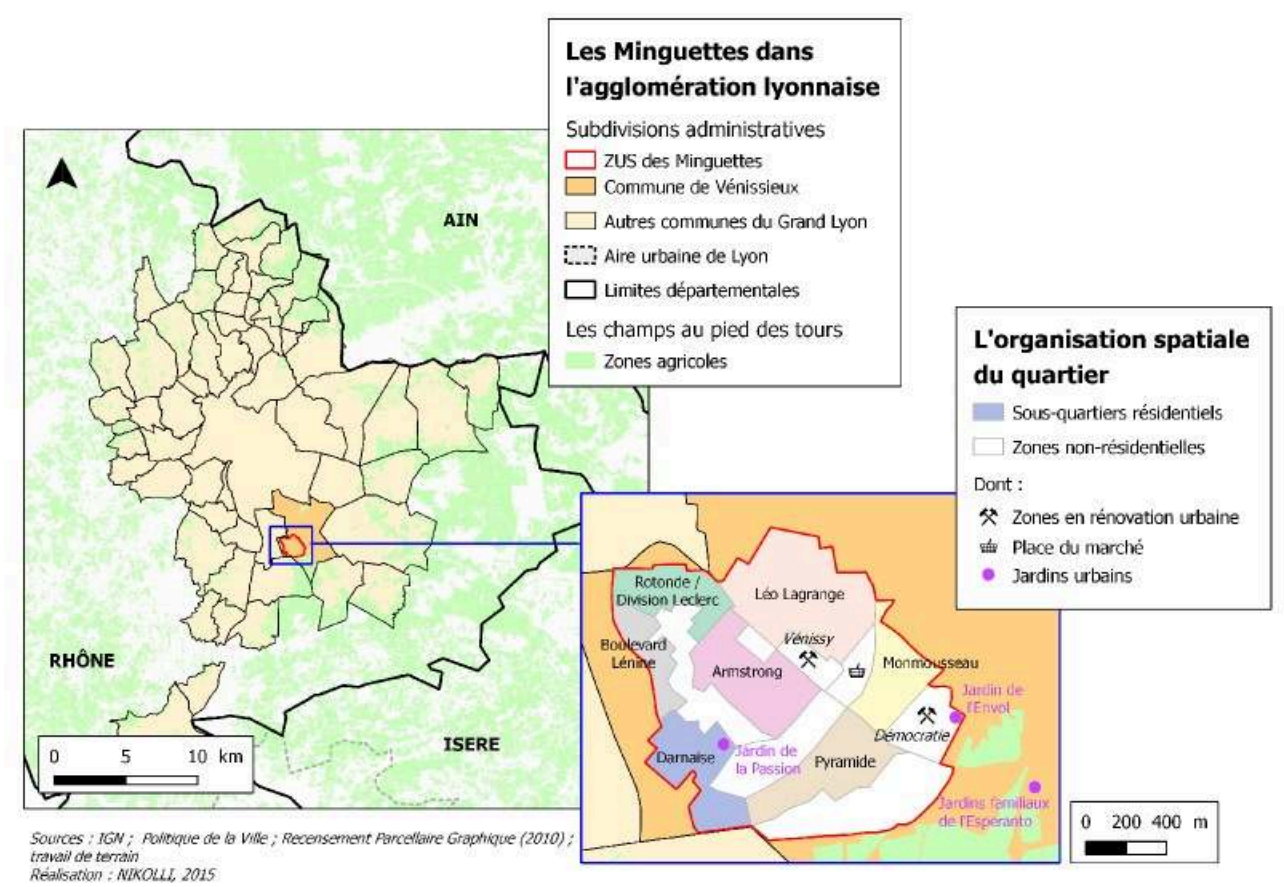

Dans ce quartier, l'offre alimentaire commerciale est assez pauvre : on n'y trouve que peu de commerces alimentaires, pour une population importante. Comme le montre la figure 2, pour 20000 habitants, le plateau ne compte que deux moyennes surfaces de proximité et deux alimentations générales, auxquelles il faut ajouter quelques commerces en pied de tours (boulangeries, boucheries, snacks). Ainsi, en moyenne, un commerce alimentaire dessert près de 1300 habitants aux Minguettes, contre 750 pour la commune de Vénissieux dans son ensemble ou 510 pour la commune de Lyon (Base permanente des équipements, Insee, 2012). Face à cette faiblesse de l'offre commerciale alimentaire, l'écrasante majorité des habitants interrogés fréquentent des hypermarchés extérieurs au quartier, pour $66 \%$ en voiture et $47 \%$ en transport en commun (Nikolli, 2014). Faiblesse de l'offre locale en produits alimentaires et déplacements massifs vers des grandes surfaces éloignées du quartier induisent la tentation de décrire le quartier des Minguettes comme un désert alimentaire, suivant la 
terminologie anglo-saxonne (Cummins et Macintyre, 2002). Cette notion désigne des espaces où l'on ne trouve pas de nourriture de bonne qualité nutritionnelle dans le commerce de proximité et où, en outre, la population est plus pauvre que la moyenne, et souvent non motorisée, ce qui contraint d'autant plus l'accès à un approvisionnement alimentaire fréquent et diversifié.

Figure 2. Une offre alimentaire peu étoffée...

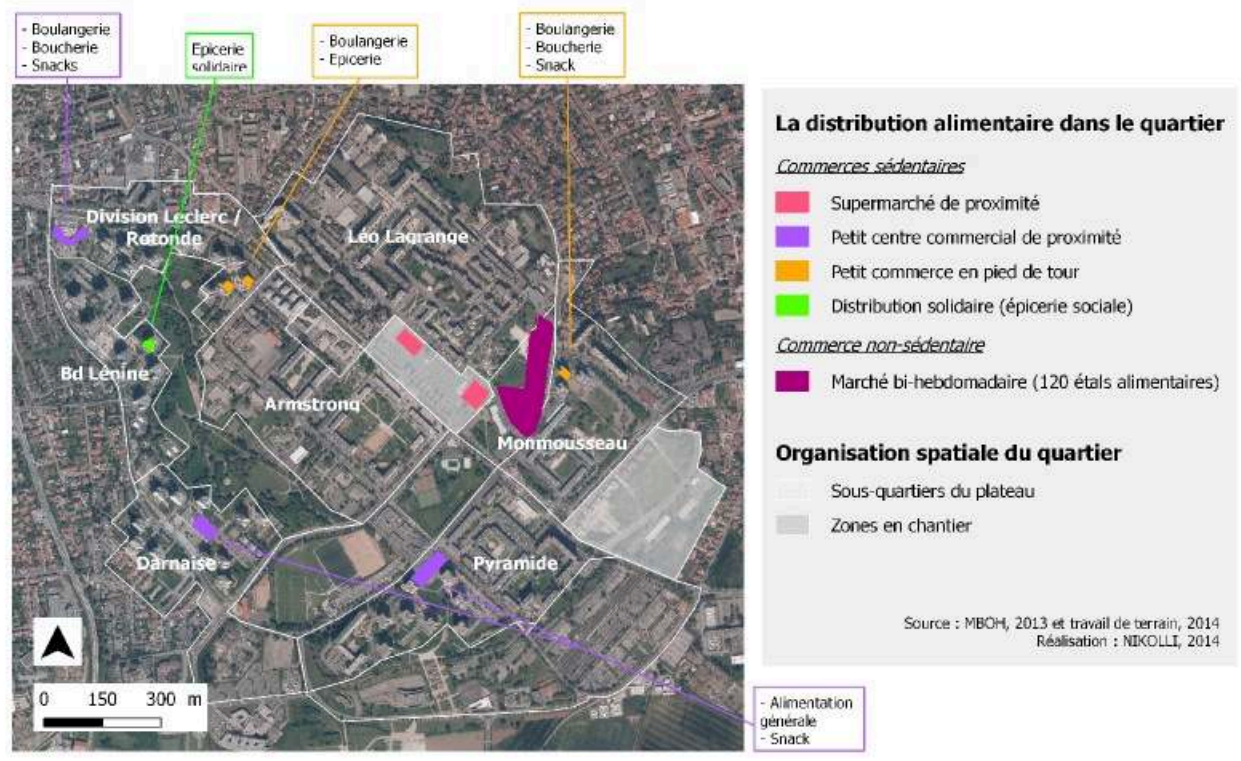

8 Mais une nuance doit être apportée. En effet, le quartier dispose d'un grand marché bihebdomadaire qui complète l'offre alimentaire en termes d'accès aux fruits et aux légumes frais, peu présents en quantité et en qualité dans les autres commerces du plateau. Ce marché s'étend sur près de 2 ha et compte jusqu'à 120 forains en alimentaire, parmi lesquels près de la moitié des étals vendent des fruits et légumes (figure 3). Il est intensément fréquenté par les habitants : $75 \%$ des habitants interrogés s'y rendent, et $70 \%$ d'entre eux au moins une fois par semaine (Nikolli, 2014). En ce sens, qualifier les Minguettes de désert alimentaire n'apparaît pas très pertinent ${ }^{1}$. 
Figure 3. ... malgré la présence d'un grand marché de plein-vent

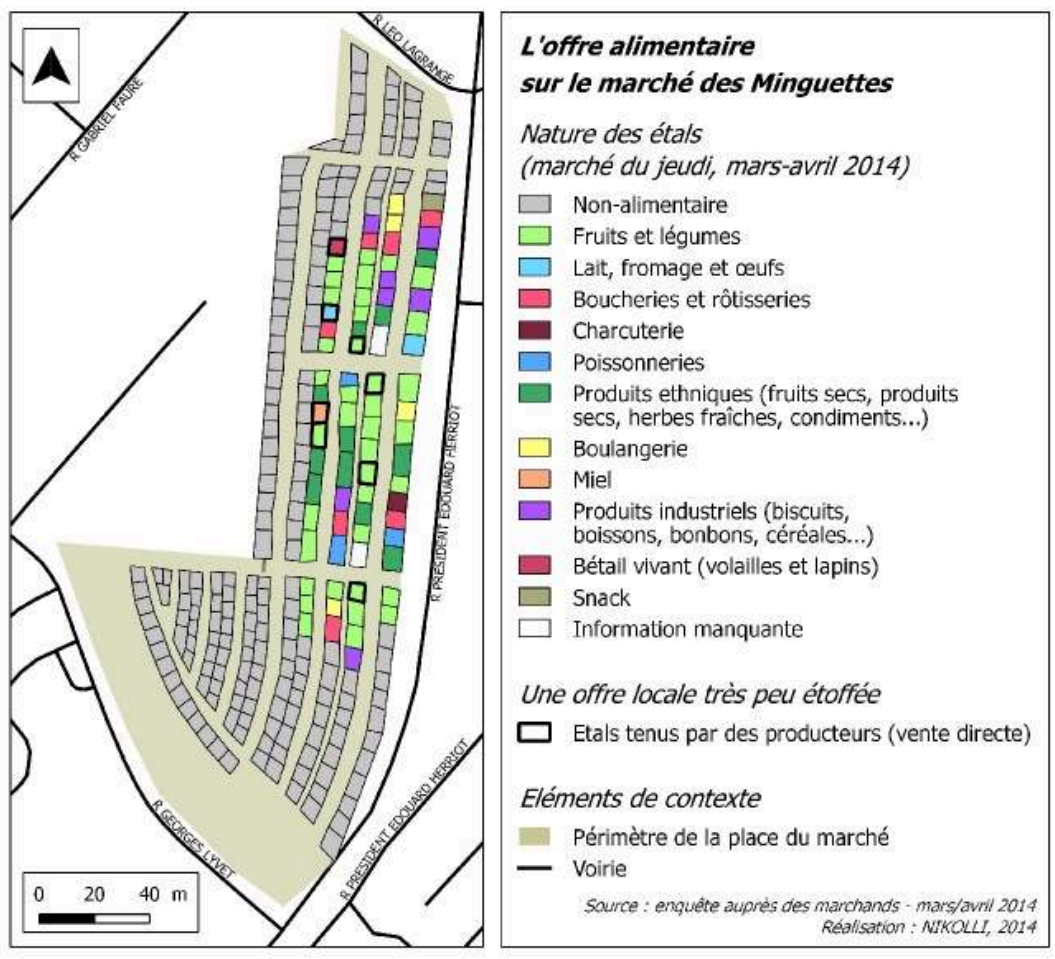

9 Néanmoins, dans les moyennes surfaces du secteur Vénissy ou sur le marché (qui compte 84 \% de revendeurs), l'offre est dominée par les circuits longs. À l'exception de quelques producteurs vendant en direct sur le marché, qui sont repérés sur la figure 3 , il n'y a aucun accès aux produits de l'agriculture locale dans le quartier. Si ce dernier ne constitue qu'un semi-désert alimentaire, il n'en demeure pas moins un désert du circuit court, au sens où il est presque impossible d'y acheter ou d'y obtenir des produits issus de l'agriculture locale, de quelque façon que ce soit (figure 4) : ni par les commerces conventionnels, ni par les structures collectives qui ailleurs commencent à faire appel à l'approvisionnement local (Darly, 2013), ni par l'aide alimentaire. Même les trois jardins collectifs du quartier ${ }^{2}$ ne constituent qu'un mince palliatif en termes d'approvisionnement local, dans la mesure où ils ne sont fréquentés que par environ 70 jardiniers. Parmi eux, seulement un petit nombre vit dans le quartier, les autres venant du reste de la commune. 
Figure 4. Un paysage alimentaire déconnecté de l'agriculture de proximité

\begin{tabular}{|c|c|c|c|}
\hline \multicolumn{2}{|c|}{$\begin{array}{c}\text { Moyen d'accès } \\
\text { potentiel à des produits } \\
\text { locaux }\end{array}$} & $\begin{array}{l}\text { Estimation du public } \\
\text { touché }\end{array}$ & Quel impact sur les paysages alimentaires? \\
\hline \multicolumn{4}{|c|}{ Circuits courts } \\
\hline \multicolumn{2}{|c|}{ Marché des Minguettes } & $\begin{array}{l}\text { Fréquenté par } 75 \% \text { de } \\
\text { l'échantillon }\end{array}$ & $\begin{array}{l}\text { Accès effectif mais très limité aux produits de } \\
\text { l'agriculture locale (environ } 10 \% \text { d'offre } \\
\text { alimentaire locale sur le marché) }\end{array}$ \\
\hline \multirow{2}{*}{$\begin{array}{c}\text { Système } \\
\text { de } \\
\text { paniers }\end{array}$} & Alterconso & $\begin{array}{l}\text { Environ } 30 \text { adhérents } \\
\text { pour la commune }{ }^{(2)}\end{array}$ & $\begin{array}{l}\text { Très peu de public touché dans le quartier des } \\
\text { Minguettes }\end{array}$ \\
\hline & $\begin{array}{l}\text { Interne à } \\
\text { Bioforce* }\end{array}$ & $\begin{array}{l}\text { Deux expériences qui ne } \\
\text { sont plus d'actualité( }{ }^{(2)}\end{array}$ & Public interne, pas d'ouverture sur le quartier \\
\hline \multicolumn{4}{|c|}{ Approvisionnement local de l'aide alimentaire } \\
\hline \multicolumn{2}{|c|}{ Épicerie solidaire } & $\begin{array}{l}174 \text { familles } \\
\text { (janvier 2014) }^{(2)}\end{array}$ & $\begin{array}{l}\text { Un projet d'approvisionnement local en partenariat } \\
\text { avec des producteurs, en cours, pas encore effectif }\end{array}$ \\
\hline \multicolumn{2}{|c|}{ Restos du cœur } & $\begin{array}{l}1230 \text { familles (hiver } \\
2013-2014)^{(4)}\end{array}$ & Pas d'accès aux produits de l'agriculture locale \\
\hline \multicolumn{2}{|c|}{ Secours populaire } & 450 familles $(2014)^{(2)}$ & Approvisionnement local ponctuel (sur un produit) \\
\hline \multicolumn{4}{|c|}{ Approvisionnement local de la restauration collective } \\
\hline \multicolumn{2}{|c|}{ Restauration collective } & 4000 repas/jour ${ }^{(2)}$ & Approvisionnement local ponctuel (sur un produit) \\
\hline \multicolumn{4}{|c|}{ Offre locale dans le commerce conventionnel } \\
\hline \multicolumn{2}{|c|}{$\begin{array}{c}\text { Commerces } \\
\text { alimentaires du plateau }\end{array}$} & $\begin{array}{l}\text { Fréquentés par } 62 \% \text { de } \\
\text { l'échantillon }^{(1)}\end{array}$ & Pas d'accès aux produits de l'agriculture locale \\
\hline \multicolumn{4}{|c|}{ Jardins urbains } \\
\hline \multicolumn{2}{|c|}{$\begin{array}{l}\text { Jardins partagés et } \\
\text { jardins familiaux }\end{array}$} & $\begin{array}{l}480 \text { personnes à l'échelle } \\
\text { de la commune }{ }^{(3)}\end{array}$ & $\begin{array}{l}\text { Autoproduction alimentaire effective mais accès } \\
\text { aux jardins très limité en termes de public }\end{array}$ \\
\hline
\end{tabular}

Sources: (1) Questionnaire aux habitants, Nikolli, 2014 ; (2) Entretiens, Nikolli, 2014 ; (3) Site de l'association Le Passe-Jardin ; (4) Site des Restos du cœur du Rhône.

Bioforce est un établissement d'enseignement supérieur privé qui forme aux métiers de l'humanitaire et du développement local et dont les locaux se situent aux Minguettes, dans le secteur Darnaise.

10 Ainsi, Vénissieux se trouve à l'écart des projets de développement d'une offre alimentaire locale, contrairement à d'autres quartiers défavorisés de l'agglomération lyonnaise qui voient se déployer des initiatives spécifiques en matière de circuits courts (figure 5). 
Figure 5. Vénissieux, à l'écart des initiatives de circuits courts

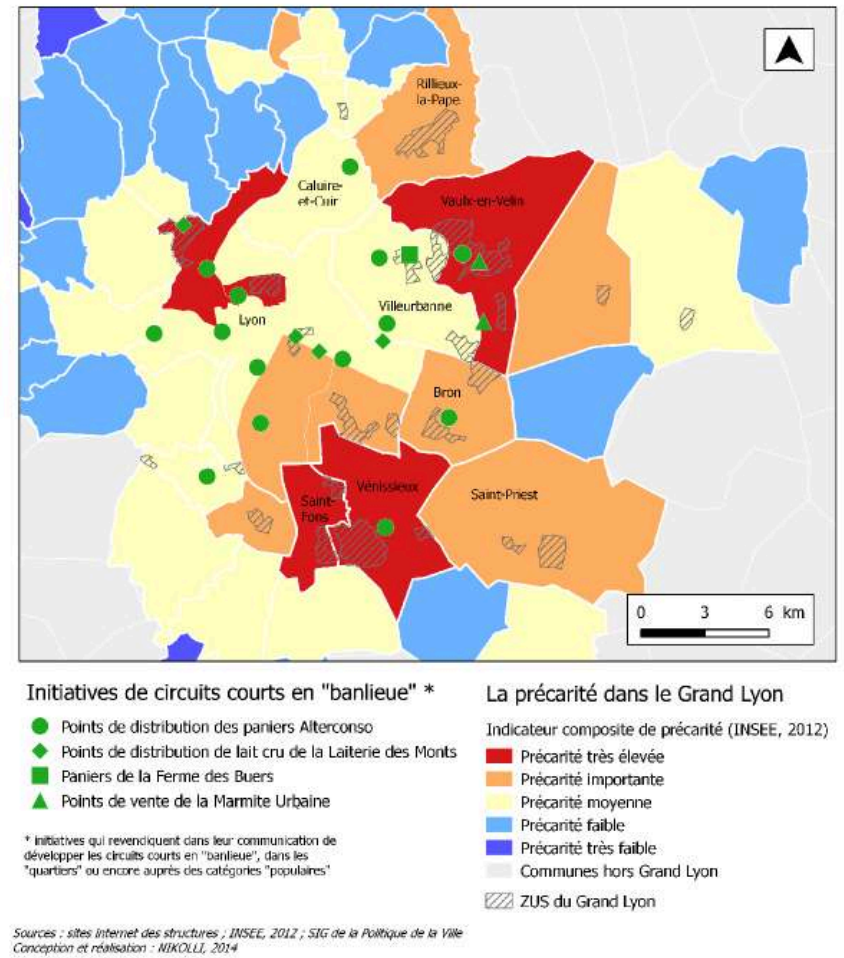

11 L'offre alimentaire du quartier des Minguettes apparaît donc non seulement peu étoffée, mais également déconnectée de l'agriculture de proximité. Or, ce constat apparaît paradoxal au regard du contexte agricole local. En effet, l'agglomération lyonnaise est entourée d'une agriculture importante (figure 1). Cette agriculture est diversifiée : à l'Ouest, on trouve de petites exploitations tournées vers la polyculture et le polyélevage, et à l'Est de plus grandes exploitations, spécialisées dans les grandes cultures. À grande échelle, on peut d'ailleurs remarquer que le quartier des Minguettes se situe à proximité immédiate des espaces agricoles (figure 1), puisque le plateau domine une vaste plaine céréalière. Il faut également souligner que cette agriculture, notamment à l'Ouest de l'agglomération, est orientée de longue date vers les circuits courts, que ce soit sous leur forme traditionnelle (marchés notamment) ou selon les modalités apparues à partir des années 2000 (première Amap lyonnaise en 2004 par exemple). Le département du Rhône est ainsi le premier de Rhône-Alpes pour la place accordée aux circuits courts, avec $42 \%$ des exploitations agricoles qui en pratiquent au moins un (Agreste, 2012). Ainsi, la déconnexion entre le quartier et l'agriculture proche interroge: dans un contexte à la fois local et national de valorisation importante des liens villes/campagnes, que savent les populations des Minguettes de leurs espaces agricoles proches? Quels paysages alimentaires trouve-t-on dans ce quartier, et quelle place l'agriculture de proximité y tient-elle? 


\section{Un protocole double pour cerner les représentations qu'ont les différents acteurs de leur offre alimentaire et agricole de proximité}

12 L'offre alimentaire du quartier des Minguettes laisse donc apparaître un paysage agroalimentaire que l'on peut, d'un point de vue externe, qualifier d'«aride». Mais dans la mesure où le paysage est surtout lié aux représentations et aux perceptions, on ne peut prétendre observer, dans un quartier de 20000 habitants encadrés par de multiples structures, un seul paysage alimentaire. On suppose donc qu'il existe des paysages alimentaires. Pour les cerner, un protocole a été mené en deux temps parallèles, qui correspondent à la fois à deux types d'expérience de recherche et à deux types de méthodologie autour des représentations de l'agriculture locale.

Des enquêtes et des entretiens ont d'abord été menés auprès de différents acteurs des Minguettes. Faire appel aux représentations des différents acteurs de ce quartier permet de dépasser les clichés habituellement véhiculés sur les espaces de grands ensembles, ou du moins de confronter ces clichés aux espaces vécus de leurs habitants et de leurs acteurs. La manière dont les populations défavorisées appréhendent leur quotidien alimentaire, suivant leurs différentes caractéristiques (genre, catégorie socioprofessionnelle, origine géographique...) éclaire la compréhension de leurs pratiques dans leur quartier. De même, le regard des acteurs publics et privés, opérant de façon directe ou indirecte sur les dispositifs alimentaires, donne à voir le rôle des représentations dans leur mise en œuvre.

Trois types d'acteurs ont été convoqués :

- 47 questionnaires ont été passés aux habitants du quartier via diverses structures associatives afin de cerner les habitudes d'approvisionnement et de consommation alimentaires et d'évaluer le degré de connaissance du tissu agricole local. Ils offrent un échantillon plutôt féminin ( $83 \%$ de femmes et $17 \%$ d'hommes) où les plus de 40 ans sont majoritaires. Les personnes interrogées nous permettent de comprendre les pratiques familiales ( $83 \%$ issues de familles) des habitants vivant dans le quartier depuis longtemps ( $87 \%$ depuis plus de 8 ans, moyenne de l'échantillon 20 ans).

- En même temps, une série d'entretiens a été menée avec des acteurs associatifs et institutionnels locaux pour cerner les politiques et les actions mises en place, et les représentations qui les sous-tendent. Les acteurs institutionnels issus de l'atelier santé-ville, des services municipaux et de la restauration collective, les associations liées aux circuits courts (jardins collectifs, groupements d'achats, distribution de paniers de produits locaux) ainsi que les acteurs de l'aide alimentaire (épicerie solidaire, Restos du cœur, Secours populaire) ont ainsi été sollicités.

- Enfin, un dernier type d'acteurs a été convoqué : les agriculteurs vendant sur le marché des Minguettes et les agriculteurs vendant en circuit court ailleurs dans l'agglomération. Comprendre les choix des lieux desservis ou non par les agriculteurs, et, en creux, les représentations qu'ils se font des Minguettes apporte des éléments d'explication supplémentaires sur l'absence de circuits courts au sein du quartier.

15 En parallèle, nous avons réfléchi à une méthode pour non seulement capter les représentations qu'ont les acteurs des Minguettes de leur agriculture locale, mais aussi observer leur potentielle évolution. Comme les problèmes d'insécurité alimentaire dans les quartiers en difficulté affectent notamment les enfants et les jeunes, ces derniers 
sont devenus des cibles prioritaires des programmes de santé et d'alimentation (Pache, 2014). Nous avons donc analysé le contenu des projets éducatifs existants et la place qu'y tient, ou que pourrait y tenir, l'agriculture de proximité. Puis nous avons proposé une action auprès d'une classe de cinquième du collège Elsa-Triolet à Vénissieux, classé en dispositif d'éducation prioritaire. Il s'agit à la fois de connaitre les représentations qu'ont les enfants de leur agriculture de proximité et de les sensibiliser à cette agriculture et, plus largement, aux systèmes alimentaires. Avec deux enseignants (en histoire géographie et en SVT), nous avons ouvert une heure de projet hebdomadaire dans le cadre de l'éducation au développement durable. Le protocole s'organise autour de quatre thématiques associées à l'agriculture de proximité: la production; la transformation et la commercialisation des produits agricoles ; la nutrition ; la justice et la solidarité alimentaires. Ces modules alternent moments théoriques et ludiques, rencontre avec des acteurs locaux du monde agricole et alimentaire, et pratique d'une agriculture de proximité avec la mise en place d'un potager. Ils s'inscrivent systématiquement dans le travail d'acquisition de compétences scolaires de niveau cinquième et intègrent aussi des éléments de collecte de données de recherche. Des temps sont enfin réservés à la valorisation au sein de l'établissement, du quartier et de la commune, dans le double but de mettre en valeur le travail des élèves et de diffuser l'information acquise.

16 Avec ce protocole de recherche fondamentale et de recherche-action, nous entendons tester plusieurs critères susceptibles d'influencer les représentations des acteurs opérant dans une marge sociale : leur âge, leur genre, leur proximité avec l'agriculture, leur fonction au sein du quartier. Il s'agit en outre de repérer si des critères liés à la situation de marge sociale peuvent influencer ces représentations. Enfin, notre objectif est aussi d'imaginer des dispositifs innovants (ici, dans le domaine éducatif) pour favoriser des reconnexions entre les marges urbaines et les franges agricoles des agglomérations.

\section{Des paysages alimentaires pluriels}

17 Les premiers résultats mettent en évidence la pluralité des paysages alimentaires du quartier des Minguettes. Cette pluralité recoupe d'emblée les différents groupes interrogés (figure 6). On distinguera ainsi le paysage alimentaire du quartier tel que se le représentent les habitants (des paysages alimentaires diversifiés mais pas nécessairement déconnectés de l'agriculture de proximité), les élèves du projet en amont de la sensibilisation (des paysages alimentaires fragmentés), les observateurs exogènes (des paysages alimentaires perçus comme dévastés) et enfin les porteurs de projet dans le domaine alimentaire (des paysages alimentaires en projet et en cours de reconnexion à l'agriculture de proximité). 
Figure 6. Des paysages alimentaires pluriels en fonction des représentations des acteurs

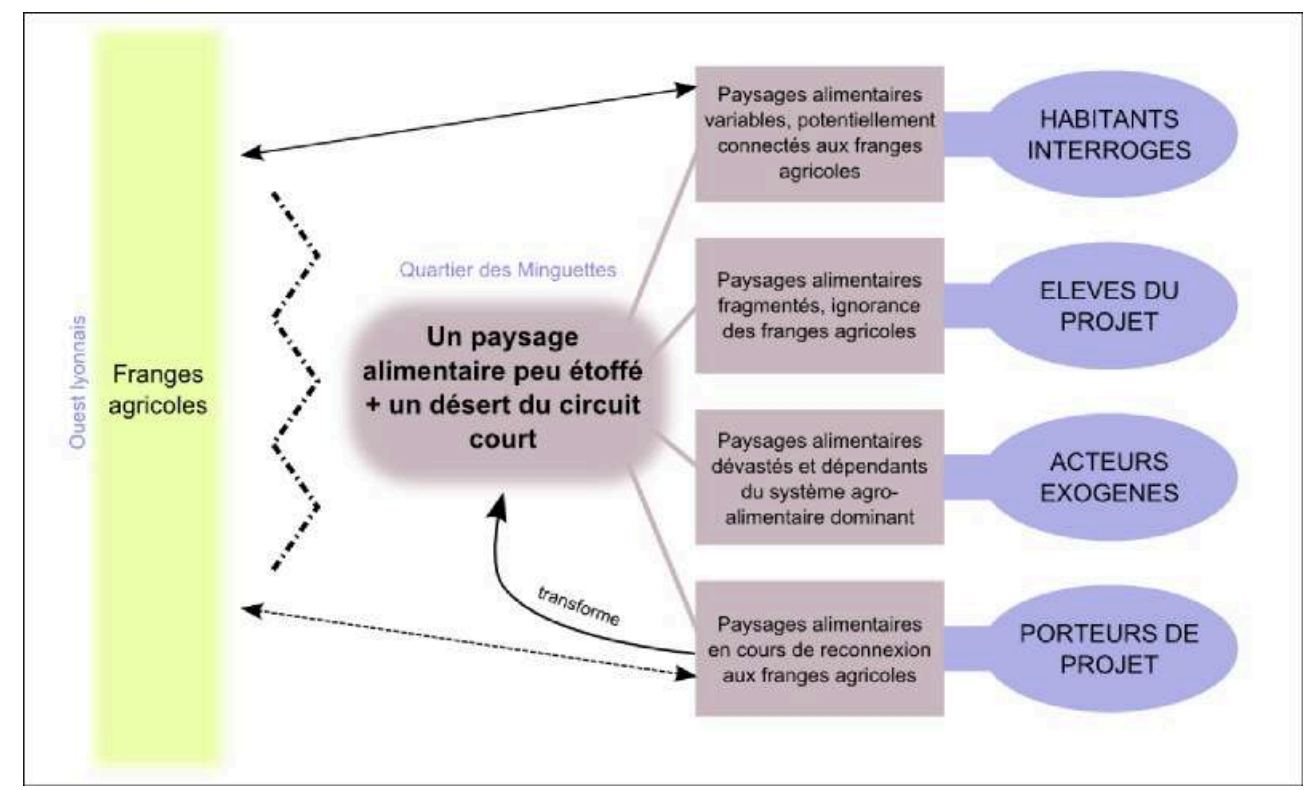

\section{Du côté des habitants, des paysages alimentaires diversifiés et relativement connectés à l'agriculture de proximité}

Les habitants du quartier décrivent des paysages alimentaires diversifiés (un constat normal puisque les "habitants " sont loin de constituer un groupe homogène), et paradoxalement moins déconnectés de l'agriculture de proximité que ce que l'état des lieux de l'offre commerciale laissait envisager. Prigent-Simonin et Hérault-Fournier (2012) soulignent que les consommateurs qui ne pratiquent pas les circuits courts ignorent souvent tout des points de vente où sont commercialisés les produits locaux. $\mathrm{Au}$ contraire, aux Minguettes, $43 \%$ des habitants interrogés font preuve d'une certaine connaissance du tissu agricole local et des opportunités d'approvisionnement qu'il représente. Dans le détail, les habitants interrogés ont à l'esprit deux types de circuits courts: les marchés $(43 \%)$ et la vente directe à la ferme $(12 \%)$, des formes traditionnelles de circuits courts qui sont aussi les plus pratiquées à l'échelle de RhôneAlpes (Agreste, 2012). Ils ont conscience de la présence d'une agriculture à proximité de leur lieu de vie, comme l'atteste une pratique qui semble régulière sur le marché des Minguettes: déposer le pain dur non consommé sous l'étal de certains producteurs pour l'alimentation de leur bétail.

Néanmoins, cette connaissance du tissu agricole local chez les habitants est segmentée.

Si près de $30 \%$ des répondants estiment qu'il y a « un peu » d'agriculture à proximité de Vénissieux, et un peu plus de $20 \%$ qu'il y en a «beaucoup», $50 \%$ de l'échantillon semblent ignorer l'existence de cette agriculture. Pourtant, le plateau des Minguettes domine une vaste plaine céréalière qui se donne à la vue ${ }^{3}$ (figure 7). On remarque que le niveau de connaissance de l'agriculture dépend surtout de l'âge des répondants: les plus de 40 ans sont surreprésentés parmi le sous-groupe caractérisé par sa connaissance du tissu agricole. Cela laisse supposer que les paysages alimentaires des habitants d'une marge varient en fonction des connaissances, des expériences et des sensibilités propres à l'égard des réalités agricoles" ${ }^{4}$. 
Figure 7. «Au dernier plan, je vois le vide, là où il y a une sorte de campagne »

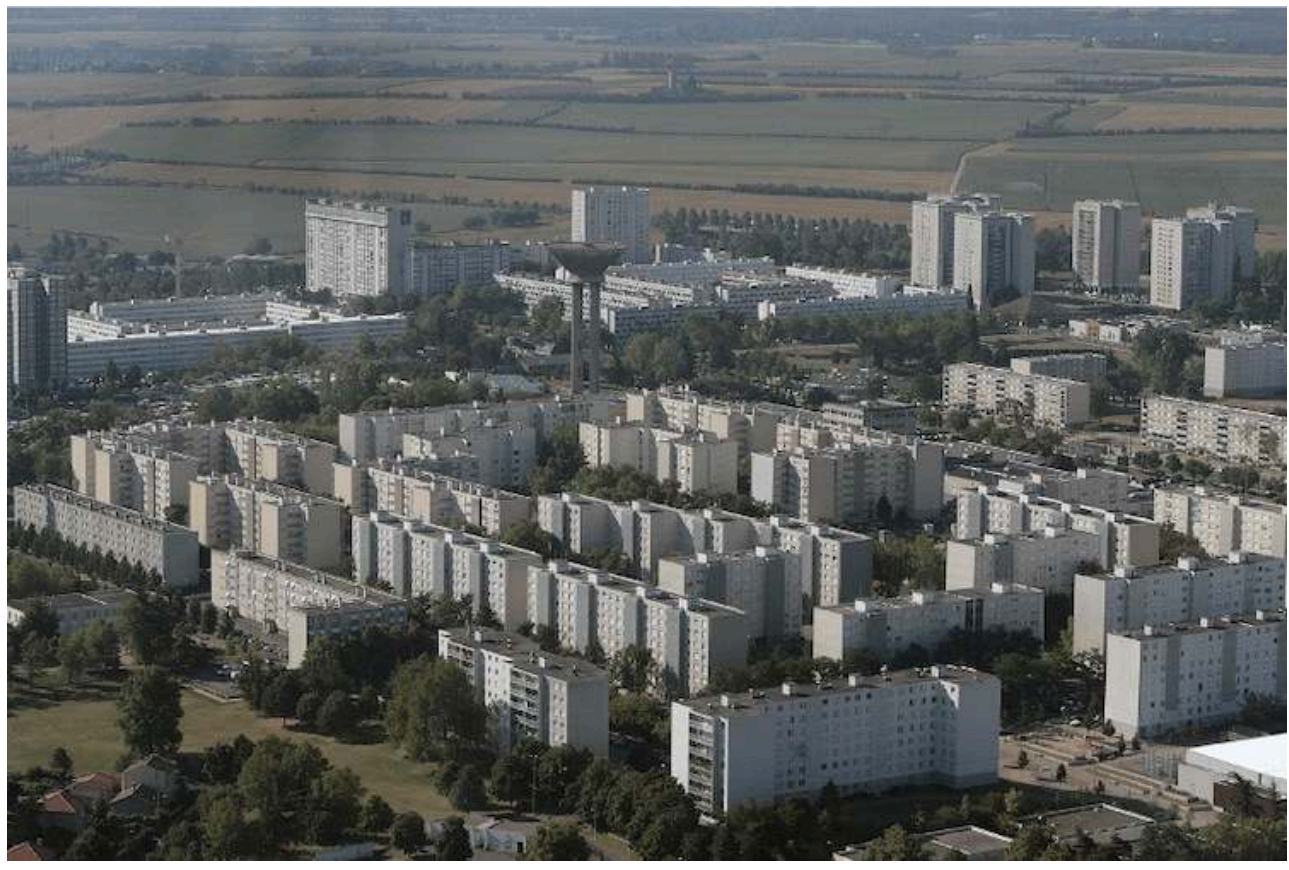

Sur cette photo, orientée au sud, on distingue nettement le quartier des Minguettes et les champs qui s'étendent en contrebas du plateau. Interrogés sur cette photo, les élèves du projet citent d'abord des éléments urbains (" tours », "bâtiments ») et les symboles des Minguettes (le château d'eau, le nom des sous-quartiers) avant de repérer « les champs », «la nature » ou encore « le vide ».

Source : Maxime Jegat, 2011.

\section{Du côté des élèves du projet, des paysages alimentaires fragmentés}

Le projet mené avec les élèves du collège Elsa-Triolet permet de consolider l'hypothèse selon laquelle l'âge contribue à la diversité des paysages alimentaires au sein d'un même quartier. Les résultats (encore provisoires) de ce projet tendent à montrer deux choses: d'une part, que les jeunes générations n'ont qu'une faible connaissance de l'agriculture et, d'autre part, qu'elles ne font pas le lien entre agriculture et alimentation.

La familiarité des adolescents avec l'agriculture est faible. Ils ne sont ainsi pas en capacité de reconnaitre les produits agricoles en cours de croissance, ce que la visite d'une exploitation maraîchère au mois d'avril, quand peu de produits sont directement reconnaissables, a permis de constater. De plus, comme le montre le diagramme cidessous (figure 8), les questionnaires proposés à 31 élèves attestent de confusions sur la fonction de l'agriculteur : ce dernier est assimilé à un simple jardinier ou à quelqu'un qui récolte, mais son métier est peu connu. 


\section{Un agriculteur, ça...}

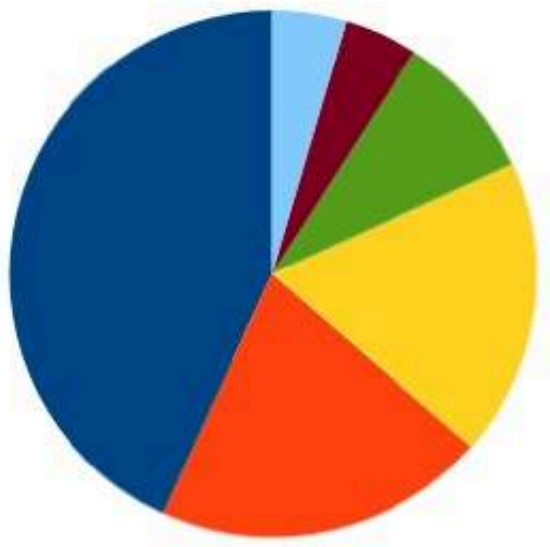

Echantillon de 31 élèves, 3 non-réponses

Question ouverte, plusteurs fonctions peuvent être èvoquées dans une seule réponse pour celle des acteurs exogènes (institutions en charge de la politique nutritionnelle, associations extérieures au quartier, agriculteurs rencontrés), qui ont une représentation pessimiste du paysage alimentaire du quartier: selon eux, il serait dévasté. Du point de vue nutritionnel comme de celui des circuits de distribution, ces acteurs ont un discours négatif sur les habitudes alimentaires - parfois avérées, parfois 
supposées - des habitants, considérés comme des victimes du système agroalimentaire dominant.

Ainsi, les acteurs en charge de la politique nutritionnelle insistent lourdement sur les problèmes de santé publique liés à l'alimentation, notamment l'obésité, qu'ils expliquent par l'influence de la publicité en lien avec un manque supposé d'esprit critique. À l'instar des travaux portant sur l'alimentation des populations précaires (Caillavet et al., 2005), ils mettent en avant une faible consommation de produits frais et une surconsommation de produits industriels transformés, riches en sucre et en graisse (grignotage, fast-food, etc.). Néanmoins, le travail de terrain a montré que ce constat - qui tient peut-être davantage de la représentation que du constat - ne va pas de soi : on peut rappeler l'intense fréquentation du marché dont l'offre en produits frais est considérable, ainsi qu'une habitude de préparation domestique des repas très marquée chez les familles, qui induit une faible consommation de produits transformés. Il ne s'agit bien sûr pas de nier l'importance des problèmes de santé publique, mais de ne pas postuler sans plus de précaution une situation de "malbouffe » généralisée. En particulier, on remarque la continuité de certaines pratiques alimentaires « ethniques » qui incluent la consommation de nombreux légumes (selon l'origine des parents ou grands-parents, principalement depuis l'Afrique du Nord et subsaharienne).

Les associations œuvrant au développement des circuits courts dans les quartiers populaires et les agriculteurs pratiquant ces circuits de distribution se représentent, quant à eux, des quartiers populaires où les circuits longs domineraient presque fatalement, du fait de la faiblesse du pouvoir d'achat de la population et du manque de sensibilisation aux questions d'agriculture locale. Leurs discours insistent sur le problème de la concurrence entre producteurs et revendeurs sur les marchés de pleinvent: les populations en situation de précarité rechercheraient des prix bas et se tourneraient systématiquement vers les revendeurs. De fait, les revendeurs dominent sur le marché des Minguettes et il semble que la différence producteurs/revendeurs ne soit pas claire dans l'esprit des habitants interrogés, dont $30 \%$ ne savent pas s'ils achètent à des producteurs ou à des revendeurs et dont $25 \%$ déclarent acheter aux deux types d'étals. Mais ce sont tout de même $36 \%$ qui déclarent privilégier les producteurs, pour des raisons de fraîcheur des produits, de qualité et de relation de confiance.

Les représentations exogènes, décrivant un paysage alimentaire dévasté, méritent donc d'être discutées à la lumière des résultats du protocole avec les habitants : bien que le quartier soit marqué par une forte précarité et d'importants problèmes sociaux, les paysages alimentaires qu'on y trouve ne sont pas pour autant dévastés. Ils sont divers, et pas nécessairement déconnectés des réalités agricoles locales, ni des règles nutritionnelles de base. Un constat que les initiatives mises en œuvre pourraient intégrer davantage.

\section{Du côté des acteurs associatifs du quartier, des paysages alimentaires en projet}

Si les paysages alimentaires du quartier varient en fonction de la façon dont se les représentent les différents acteurs, soulignons qu'ils sont également évolutifs. Ainsi, en s'intéressant aux acteurs associatifs œuvrant dans le quartier, on découvre des paysages alimentaires en projet et en cours de reconnexion à l'agriculture de 
proximité. En effet, plusieurs de ces projets contribuent à faire évoluer les paysages alimentaires de manière très concrète.

C'est tout particulièrement le cas des jardins urbains, qui permettent dans une certaine mesure une autoproduction alimentaire par les habitants, et qui induisent une nouvelle manière de considérer l'alimentation et son processus de production. On compte aux Minguettes deux jardins partagés, auxquels on peut ajouter les jardins familiaux qui s'y trouvaient jusqu'en 2007 et qui ont été déplacés en contrebas du plateau (figure 1). Ces jardins touchent un faible nombre de personnes - de l'ordre de quelques dizaines mais ont un impact important sur la situation alimentaire des individus concernés, comme cela a pu être montré dans d'autres régions (Pourias et al., 2012). Ainsi, la production du jardin représenterait 20 à $30 \%$ de la consommation annuelle moyenne de fruits et de légumes par personne pour les deux jardins partagés, et la quasi-totalité de l'approvisionnement en fruits et légumes, du moins en pleine saison, pour les jardins familiaux ${ }^{5}$. Ces initiatives agri-urbaines font évoluer le paysage urbain en lui-même, comme on peut le voir sur la photo ci-dessous (figure 9), mais aussi les paysages alimentaires des habitants, dans la mesure où elles permettent de reconnecter de manière très directe alimentation et agriculture. Ainsi, les personnes ayant un jardin semblent plus sensibles à la qualité et à la provenance de leur alimentation : ce sont par exemple $57 \%$ des personnes ayant un jardin qui privilégient les producteurs sur le marché, contre $36 \%$ pour l'échantillon total.

Figures 9. Le jardin de la Passion, au pied des tours de la Darnaise

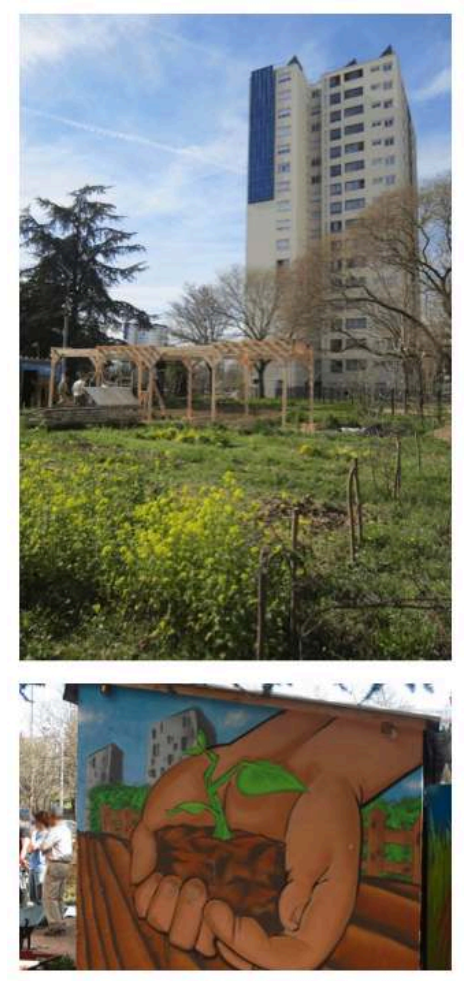

À haut : le jardin de la Passion, jardin partagé dans le secteur Darnaise. Créé en 2009 à l'initiative du bailleur social Grand Lyon Habitat, il regroupe une dizaine de jardiniers sur une surface cultivée de $800 \mathrm{~m}^{2}$; en bas : la fresque murale de la cabane à outils du jardin, peinte par des jeunes du quartier Source : Alice Nikolli, mars 2014. 
autres projets tendent à créer de nouveaux circuits d'approvisionnement pour les habitants du quartier : une association met en place des groupements d'achats et une épicerie solidaire cherche à s'approvisionner en direct auprès de producteurs locaux. Ces deux projets visent à relier le quartier et l'agriculture locale dans l'optique d'une amélioration de la situation alimentaire des familles, en leur permettant de consommer des produits frais de qualité à prix réduit.

Enfin, le projet de sensibilisation à l'agriculture mené avec les enfants du collège vise à faire évoluer leurs représentations. Pour ce faire, un potager avec une serre rudimentaire a été créé à la rentrée 2014 (figure 10) et des rencontres avec des producteurs locaux ont été organisées: l'objectif est d'associer aux connaissances théoriques un travail pratique sur l'agriculture qui permette de mieux comprendre l'activité et ses enjeux majeurs. En outre, les enfants ont fait campagne auprès d'agriculteurs pour collecter des produits frais à destination de l'épicerie solidaire : il s'agit là d'éveiller les enfants à la fois à la solidarité alimentaire et à l'importance des produits frais au sein de leur alimentation. En constatant la difficulté du personnel de l'épicerie à collecter des produits frais, ils prennent aussi conscience des situations de malnutrition que vivent certains de leurs voisins.

À la croisée de ces différents projets et des choix individuels, le paysage alimentaire des Minguettes est donc susceptible d'évoluer et de tisser de nouveaux liens avec l'agriculture de proximité.

Figure 10. Un potager pour faire évoluer les représentations

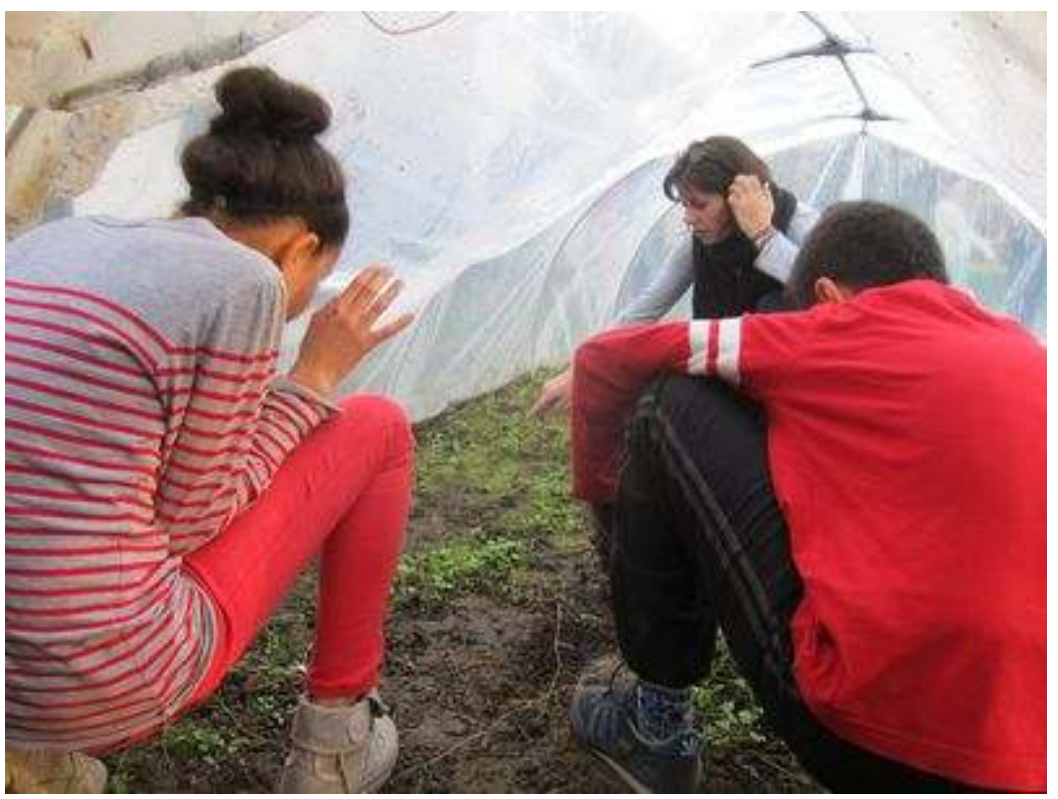

Les séances de projet avec les cinquièmes alternent entre la salle de classe et le potager, créé par les élèves en 2014-2015. Ici, l'enseignante montre aux enfants l'évolution des pousses d'épinard depuis qu'ils les ont plantées, avant de procéder à une opération de désherbage de la serre.

Source : Julie Le Gall, novembre 2014.

Ces premiers résultats invitent à développer d'autres recherches. En effet, les dimensions de l'échantillon ne permettent pas d'identifier suffisamment l'influence des caractéristiques sociales dans la construction des paysages alimentaires et, au-delà, dans les situations de connexion ou déconnexion avec l'agriculture locale. On constate 
bien que la marginalité du quartier des Minguettes contribue à donner leur caractère "dévasté » aux paysages alimentaires décrits par des acteurs exogènes, dont les agriculteurs, et donc à limiter l'implantation de circuits courts. Mais d'autres facteurs pourraient intervenir. Ainsi, si certains agriculteurs expliquent qu'ils ne se rendent pas aux Minguettes pour des questions de rentabilité, comment expliquer que d'autres, dans d'autres communes défavorisées, acceptent de participer à des initiatives sociales? Il se peut que les choix dans la gouvernance alimentaire, variables d'un quartier à un autre, d'une commune à une autre, interviennent également dans les initiatives de mise en réseau entre producteurs et consommateurs. De même, concernant les enfants, il semble pour l'heure difficile d'isoler la variable âge de la variable marginalité sociale: les enfants de douze ans, quelle que soit leur origine sociale, ont probablement le même faible niveau de connaissance concernant l'agriculture locale et l'agriculture en général. Il semble nécessaire de déployer un protocole similaire dans d'autres marges et dans d'autres types d'espaces, afin de disposer d'échantillons comparatifs et d'en extraire les variables sociales.

\section{Conclusion}

La notion de paysage alimentaire permet à la fois de diagnostiquer l'offre commerciale d'un espace donné et d'appréhender les représentations que se font différents acteurs opérant dans un même territoire des opportunités alimentaires de leur environnement proche. Appliquée au quartier des Minguettes, la notion révèle que la connexion des marges urbaines aux franges agricoles périurbaines ne va pas de soi : dans un quartier globalement déconnecté de l'agriculture de proximité, la diversité des paysages alimentaires et des degrés de connexion à l'agriculture décrits par les différents acteurs met en lumière les divergences de points de vue, et donc potentiellement de projets, à mettre en œuvre en vue d'une reconnexion. Le fait que les marges urbaines et les franges agricoles périurbaines se tournent le dos illustre une intégration différenciée des territoires au vaste mouvement, réassociant les villes et les campagnes. C'est dans cette différence (de degré, de nature) que se pose tout l'enjeu de justice alimentaire.

Mais il s'agit bien là de processus en construction : à la croisée des pratiques et des représentations, les paysages alimentaires sont susceptibles d'évoluer avec l'offre concrète et avec des projets associés aux représentations des uns et des autres. Ainsi, une opération de sensibilisation à l'agriculture peut réduire la distance mentale entre consommation et production, et entre villes et franges agricoles périurbaines: elle serait alors susceptible de diversifier les paysages alimentaires de proximité des consommateurs en les rendant plus «agri-alimentaires». De telles recherches appellent néanmoins un approfondissement. En effet, il importe de mieux comprendre l'influence des critères sociaux sur les représentations des adolescents (capital culturel, milieu familial...), de même que le rôle de leur environnement immédiat, plus ou moins urbain, plus ou moins rural. C'est en sortant des marges sociospatiales urbaines et en explorant d'autres territoires marqués par une plus ou moins grande pauvreté que nous testerons les premières conclusions présentées ici.

Les auteurs remercient vivement les autres membres du projet impliqués dans l'élaboration des résultats : Luc Merchez (ENS de Lyon), Camille Hochedez (université de Poitiers), ainsi que la direction du collège Elsa-Triolet de Vénissieux qui a autorisé la réalisation de cette recherche en 
ses murs. Ils remercient également l'entreprise Solvay pour son soutien financier obtenu par l'intermédiaire de Bruno Cosantino.

\section{BIBLIOGRAPHIE}

Agreste, « Circuits courts : Rhône-Alpes est branchée », Coup d'Æil, 2012.

Alkon, A. H., Agyeman, J., Cultivating food justice : race, class and sustainability. Food Health and the Environment, Cambridge, MIT Press, 2011, 404 p.

Allen, P., Fitzsimmons, M., Goodman, M., Warner, K., « Shifting plates in the agrifood landscape : the tectonics of alternative agrifood initiatives in California ", Journal of rural studies, vol. $19, \mathrm{n}^{\circ} 1$, 2003, p. 61-75.

Barnier, M., « Renforcer les liens entre producteurs et consommateurs - Plan d'action pour développer les circuits courts ", Paris, ministère de l'Agriculture, 2009.

Beraud-Sudreau, A., « Les politiques d'intervention dans le domaine alimentaire », extrait du «Rapport pour la promotion de l'approvisionnement local et/ou biologique de la restauration collective en Rhône-Alpes », Draaf Rhône-Alpes, AgroSup Dijon, 2010.

Block, J.-P., Scribner, R. A., De SalvoA, K. B., « Fast food, race/ethnicity, and income : A geographic analysis », American Journal of Preventive Medicine, vol. 27, n 3, 2004, p. 211-217.

Caillavet, F., Darmont, N., Lhuissier, A., Régnier, F., « L'alimentation des populations défavorisées en France. Synthèse des travaux dans les domaines économique, sociologique et nutritionnel », «Travaux de l'observatoire national de la pauvreté et de l'exclusion sociale », Observatoire national de la pauvreté et de l'exclusion sociale, 2005.

Chaffote, L., Chiffoleau, Y., " Vente directe et circuits courts : évaluations, définitions et typologie », Les Cahiers de l'Observatoire CROC, $\mathrm{n}^{\circ}$ 1, 2007.

Cummins, S., Macintyre, S., « A systematic study of an urban foodscape : the price and availability of food in greater Glasgow", Urban Studies, vol. 39, 2002.

Darly, S., « Relocaliser pour mieux négocier ou négocier pour mieux relocaliser ? Négociations et compromis pour la construction des réseaux locaux de l'approvisionnement des cantines ", SudOuest européen, $\mathrm{n}^{\circ}$ 35, 2013, p. 31-42.

Dixon, J., Isaacs, B., « Why sustainable and "nutritionary correct" food is not on the agenda : Western Sydney, the moral arts of everyday life and public policy », Food Policy, vol. 43, 2013, p. 67-76.

Gottlieb, R. et Joshi, A., Food justice, Cambridge, MIT Press, 2010.

Jouve, A.-M., Padilla, M., « Les agricultures périurbaines méditerranéennes à l'épreuve de la multifonctionnalité : comment fournir aux villes une nourriture et des paysages de qualité ?», Cahiers Agricultures, volume 16, $\mathrm{n}^{\circ}$ 4, 2007. 
Kelly, B., Cretikos, M., Rogers, K., King, L., « The commercial food landscape : outdoor food advertising around primary schools in Australia ", Australian and New Zealand Journal of Public Health, vol. 32, $\mathrm{n}^{\circ}$ 6, 2008, p. 522-528.

Larsen, K., Gilliland, J., « A farmers' market in a food desert : Evaluating impacts on the price and availability of healthy food ", Health \& Place, vol. 15, nº 4, 2009, p. 1158-1162.

Mundler, P., « Le prix des paniers est-il un frein à l'ouverture sociale des Amap ? Une analyse des prix dans sept Amap de la région Rhône-Alpes ", Économie rurale, n 336, 2013, p. 3-19.

Nikolli, A., «L'accès aux produits de l'agriculture locale dans un quartier de banlieue lyonnaise : l'exemple des Minguettes (Vénissieux) », mémoire de master 1, ENS de Lyon, 2014, 150 p.

Pache, A., L'Alimentation à l'école, pour une géographie renouvelée, Rennes, Presses universitaires de Rennes, 2014, 359 p.

Paddeu, F., « L'agriculture urbaine dans les quartiers défavorisés de la métropole New-Yorkaise : la justice alimentaire à l'épreuve de la justice sociale ", VertigO. La revue électronique en sciences de l'environnement, vol. 12, $\mathrm{n}^{\circ}$ 2, 2012, URL : http://vertigo.revues.org/12686.

Pourias, J., Daniel, A.-C., Aubry, C., « La fonction alimentaire des jardins associatifs urbains en question », Pour, vol. 215-216, n 3, 2012, p. 333-347.

Prigent-Simonin, A. H. et Hérault-Fournier, C., Au plus près de l'assiette. Pérenniser les circuits courts alimentaires, Versailles et Paris, Quae et Educagri, coll. « Sciences en partage », 2012, 261 p.

Rödiger, M., Hamm, U., « How are organic food prices affecting consumer behaviour ? À review », Food Quality and Preference, vol. 43, 2015, p. 10-20.

\section{NOTES}

1. En outre, le contexte français, différent des contextes étasunien et canadien dans lesquels la notion de food desert a été développée, rend difficile la stricte transposition de la notion : le zonage fonctionnel et la suburbanisation du commerce sont moins marqués en France qu'outreAtlantique, tandis que la géographie sociale des villes est inversée (si l'on brosse cette géographie à grands traits, le gradient de pauvreté augmente du centre vers les périphéries en France, tandis qu'il diminue aux États-Unis et au Canada).

2. On recense dans le quartier trois jardins collectifs de nature différente: un jardin partagé d'habitants, un jardin d'insertion sociale et des jardins familiaux.

3. On peut néanmoins supposer que l'agriculture locale serait mieux identifiée dans l'Ouest lyonnais, connu pour être une région agricole vendant en direct dans l'agglomération. À l'inverse, l'Est de l'agglomération est plus connu pour ses activités industrielles, tandis que son agriculture, orientée sur les céréales et les oléagineuses, ne pratique pas les circuits courts.

4. En ce sens, on note que les enfants connaissent souvent très bien la réalité agricole du pays d'origine de leur famille (Maroc, Algérie principalement) où ils ont de la famille qui pratique encore cette petite agriculture. La connaissance des réalités agricoles mérite donc une appréhension à plusieurs échelles : le paysage alimentaire local des enfants n'intègre peut-être pas l'agriculture, ce qui n'est pas le cas de leur « autre " paysage de proximité lié à leur famille.

5. Sources : pour les jardins partagés, production annuelle moyenne par jardinier (communiquée lors des entretiens avec les porteurs de projet) ramenée à la consommation annuelle moyenne par personne en France (186,7 kg d'après France AgriMer en 2008) ; pour les jardins familiaux, constat empirique du président de l'association. 


\section{RÉSUMÉS}

Si un engouement croissant pour les produits locaux se fait sentir, force est de constater que ce changement dans la consommation alimentaire concerne surtout les quartiers centraux des villes et les populations à fort capital social et culturel. Le travail de recherche dont est issu cet article vise à questionner ce constat: les marges urbaines et leurs habitants sont-ils déconnectés des franges agricoles qui fournissent à la ville des produits alimentaires locaux et, si oui, pourquoi ? En s'appuyant sur la notion exploratoire de paysage alimentaire, nous avons voulu tester l'importance des représentations que les habitants des espaces urbains défavorisés ont des franges agricoles et des opportunités d'approvisionnement alimentaire qui en sont issues. Dans le cadre d'un travail de terrain et d'un projet en milieu scolaire mené dans le quartier des Minguettes (Vénissieux, agglomération lyonnaise), nous avons cherché à cerner les paysages alimentaires de plusieurs catégories d'acteurs et leur influence sur les modes de consommation alimentaire des habitants.

Although there is an increasing demand for local produce this change in food consumption concerns principally people living in city centres with high social and cultural capital. The research presented in this article seeks to answer the following question: Are suburban areas and their inhabitants disconnected from the agricultural fringe areas which supply the city with local food products and, if so, why? Based on the exploratory notion of the food landscape, we have sought to test the importance of the representations the inhabitants of disadvantaged urban areas have of the agricultural fringes and of the opportunities they provide as food sources. Within the framework of a field study and of a school project conducted in the Minguettes district (in Vénissieux in the agglomeration of Lyon), we have sought to identify the food landscapes of several types of stakeholders and their influence on the modes of food consumption of the inhabitants.

\section{INDEX}

Mots-clés : paysage alimentaire, justice alimentaire, circuits courts, marges urbaines, agriculture de proximité

Keywords : food landscape, food equity, short cycles, urban fringes, local agriculture

\section{AUTEURS}

\section{ALICE NIKOLLI}

Alice Nikolli est élève en master (université de Lyon, École normale supérieure de Lyon, UMR 5600 EVS, équipe Biogéophile), elle est agrégée de géographie. alice.nikolli[at]ens-lyon[dot]fr

\section{JULIE LE GALL}

Julie Le Gall est maître de conférences en géographie à l'université de Lyon, École normale supérieure de Lyon, UMR 5600 EVS, équipe Biogéophile.

julie.legall[at]ens-lyon[dot]fr 


\section{MYRIAM LAVAL}

Myriam Laval est professeure d'histoire et géographie au collège Elsa-Triolet à Vénissieux, elle est agrégée de géographie.

lavalmyriam[at]yahoo[dot]fr 